\title{
La veille stratégique, un outil pour favoriser l'innovation au canada
}

\author{
François Brouard ${ }^{1}$ \\ Eric Sprott School of Business, Carleton University
}

\section{Introduction}

Face aux défis posés par la complexité grandissante, les changements rapides, les incertitudes de plus en plus nombreuses, diverses actions doivent être prises et le sont pour améliorer le fonctionnement des organisations et l'atteinte de leurs objectifs à l'ère de l'information et de l'économie du savoir. Comme l'adaptation aux changements de l'environnement est une nécessité de tous les instants pour faire face à une concurrence de plus en plus vive au niveau international, l'identification des changements le plus efficacement possible est un problème de gestion méritant une étude plus approfondie.

Les nouvelles réalités économiques nécessitent de nouveaux modes d'apprentissage afin de faire ressortir les connaissances utiles aux entreprises pour affronter l'environnement changeant. Divers leviers existent pour améliorer l'apprentissage collectif d'une organisation. L'un de ces leviers, la veille stratégique, permet de créer de l'information structurante. La veille stratégique peut se définir comme un processus informationnel par lequel une organisation se met à l'écoute de son environnement pour décider et agir dans la poursuite de ses objectifs. Ainsi, la veille stratégique réalisée par les organisations leur permet de favoriser l'innovation.

L'objectif de cet article est de définir la veille stratégique, de présenter les composantes de celle-ci, c'est-à-dire la veille technologique, la veille commerciale, la veille concurrentielle et la veille sociétale, de souligner son rôle, de décrire le processus de veille stratégique et de faire état des travaux de recherche effectués dans le domaine au Canada. Pour conclure, des pistes de recherche et d'action sont esquissées afin d'inciter les décideurs gouvernementaux et ceux du monde des affaires à prendre aujourd'hui des moyens pour construire l'entreprise de demain. La suite du présent texte se divise en trois grandes parties. La première présente une description de la veille stratégique. La deuxième fait un survol des travaux de recherche portant sur la veille stratégique. La troisième identifie des pistes de recherche et d'action.

\section{Description de la veille}

\section{Définition de la veille stratégique}

Il y a lieu de souligner la prolifération de termes et la confusion terminologique, tant en français qu'en anglais, quand il est question de décrire la veille stratégique. Cette confusion provient aussi de

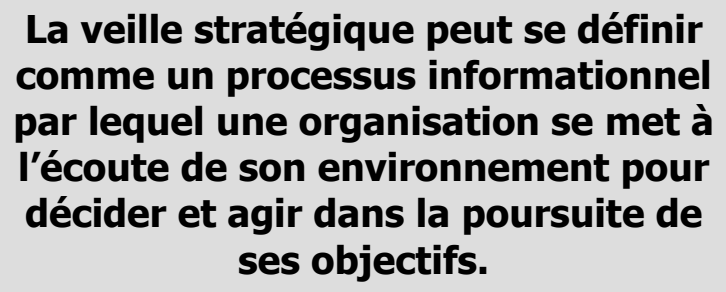

La veille stratégique peut se définir comme un processus informationnel par lequel une organisation se met à l'écoute de son environnement pour décider et agir dans la poursuite de ses objectifs. l'utilisation de termes qui représentent simultanément deux éléments, soit le processus de la veille et le résultat de ce processus. L'expression « veille stratégique » est suggérée pour décrire le processus, et l'expression "intelligence de l'entreprise » est suggérée pour décrire le résultat final de ce processus; les expressions anglaises environmental scanning et business intelligence sont utilisées pour décrire respectivement le processus et le résultat.

Afin de mieux cerner et définir la veille stratégique, certaines caractéristiques ou dimensions de ce concept sont retenues : la finalité, l'objet, le processus et l'environnement. La finalité correspond à l'objectif 
poursuivi et est liée à l'action et aux décisions à prendre. L'objet représente les changements sous observation et sous analyse. Des pôles ou des sujets particuliers constituent l'objet du processus de la veille stratégique. Il peut s'agir d'identifier les changements d'un ou de plusieurs pôles tels la technologie, les produits et procédés, les concurrents, les clients, les fournisseurs et les tendances lourdes de la société. Le processus correspond à la transformation nécessaire pour atteindre les objectifs poursuivis.

Une méthodologie est généralement associée au processus de veille stratégique. L'environnement, tant externe qu'interne, comprend l'ensemble des facteurs qui entourent le cycle de la veille stratégique.

S'appuyant sur les quatre caractéristiques identifiées, le concept de veille stratégique se définit comme un processus informationnel par lequel une organisation se met à l'écoute de son environnement pour décider et agir dans la poursuite de ses objectifs.

\section{Types et rôles de la veille}

Peu importe sous quel angle la veille stratégique est abordée, l'enjeu central demeure la survie de l'entreprise qui est menacée par l'incertitude créée dans l'environnement. La prise de décisions pour assurer cette survie est au cœur des activités de veille. La veille sert principalement quatre fonctions: 1) appuyer la prise de décisions stratégiques, 2) servir d'avertissement (radar) pour les occasions et les menaces, 3) évaluer les compétiteurs et les suivre, 4) appuyer la planification stratégique et sa mise en œuvre. Quatre types de veille peuvent être soulignés, soit la veille technologique, la veille concurrentielle, la veille commerciale et la veille sociétale. La veille stratégique englobe l'ensemble des veilles particulières. Chaque type de veille permet de combler des besoins particuliers. Le tableau 1 illustre les quatre types de veille et leurs axes de surveillance selon les sujets privilégiés et les forces du modèle de Porter $(1982)^{2}$.

Tableau 1 - Axes de surveillance selon les différents types de veille

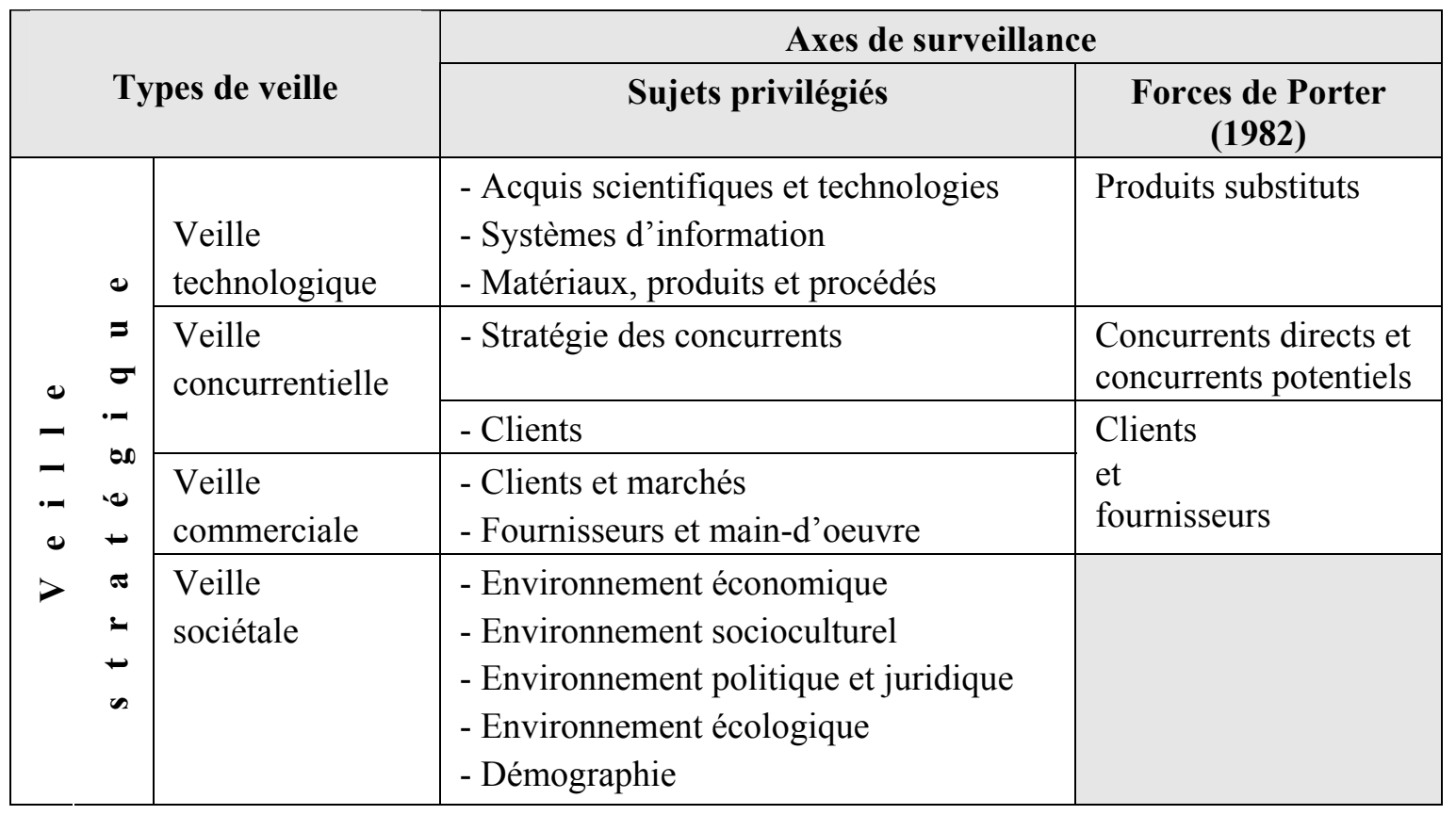

\section{Processus de la veille stratégique}

Le processus de la veille stratégique peut se décrire comme un système. Ce système se compose lui- même de sous-systèmes. Ces système et soussystèmes de la veille stratégique sont influencés par des flux d'information provenant du macroenvironnement, des acteurs et de l'organisation elle- 
même. Le macro-environnement se compose des dimensions démographique, économique, technologique, politique, juridique, écologique, géophysique et socioculturel. Les acteurs ou détenteurs d'enjeux (stakeholders) identifiés sont les clients, les fournisseurs (incluant les bailleurs de fonds), les employés, les syndicats, les partenaires, les compétiteurs, les gouvernements, les médias, les groupes de pression et les réseaux. L'environnement interne de l'organi- sation correspond à ses ressources, à sa culture, à ses stratégies, à sa direction et à sa structure. Les dimensions de l'environnement interne ont une influence sur des sous-systèmes liés aux ressources, à la culture, à la direction et à la structure de la veille. La figure 1 illustre les systèmes du processus de la veille stratégique et les influences qui les affectent.

\section{Figure 1 - Processus de la veille stratégique et flux}

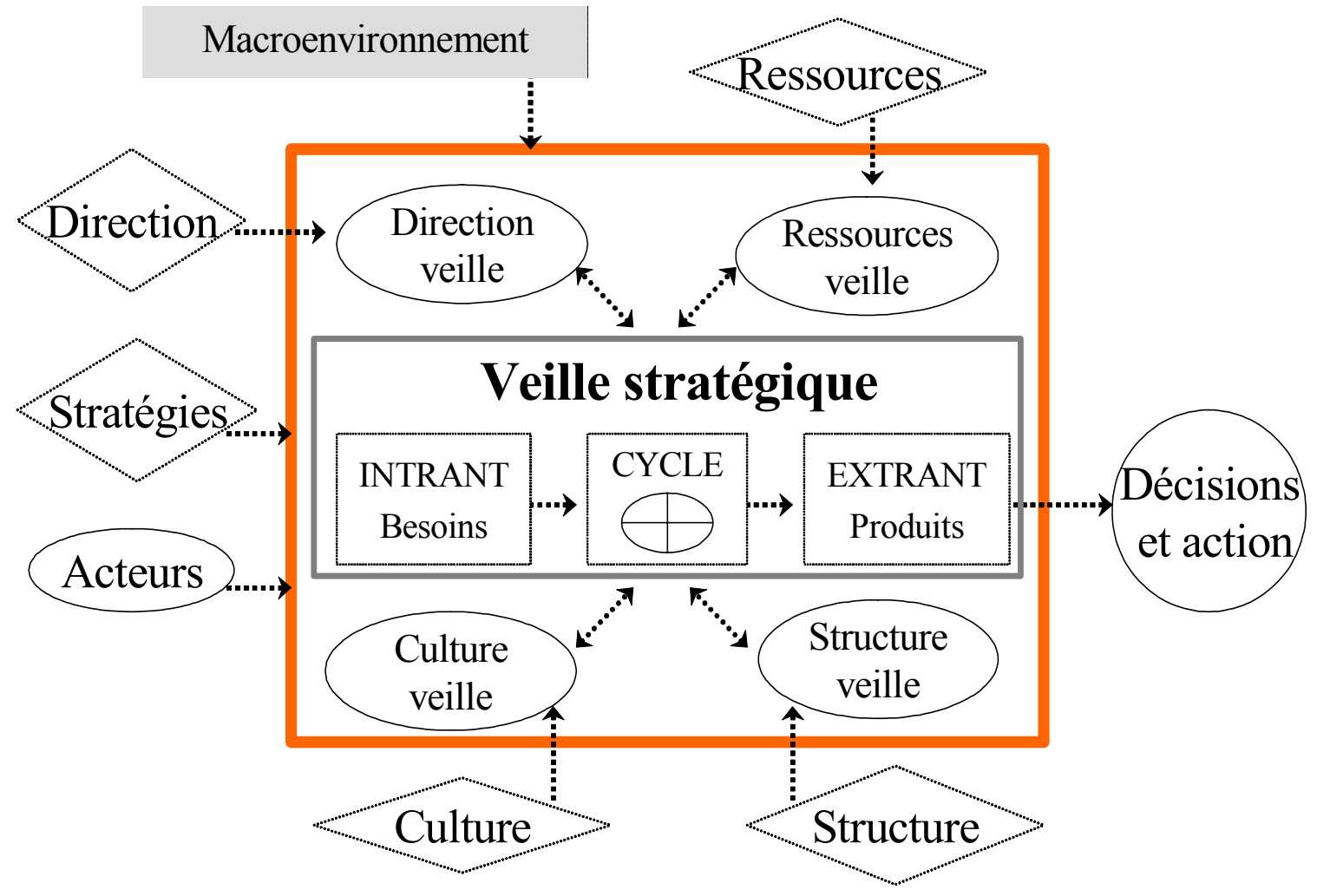

(C) 2002, François Brouard

Le système principal du processus de la veille stratégique se décompose en trois éléments, soit les intrants, le cycle et les extrants. Les intrants correspondent aux nombreux besoins manifestés par les utilisateurs. Le cycle de la veille stratégique est abordé par la plupart des auteurs, qui regroupent le processus de l'intelligence en quelques phases seulement. Il importe de souligner l'aspect continu et perpétuel du cycle qui s'apparente à une spirale, et l'aspect dynamique des différentes phases du cycle. La figure 2 illustre les quatre phases du cycle retenu, soit la planification, la collecte, l'analyse et la diffusion. Les extrants correspondent aux produits orientés vers les décisions et l'action. Selon les phases du cycle de la veille stratégique, ces produits peuvent être des données, de l'information ou des connaissances.

\section{Travaux de recherche portant sur la veille}

Il existe peu d'études portant sur les pratiques de veille stratégique au Canada. Cela peut s'expliquer par la jeunesse de ce domaine d'étude et par 
l'absence de cadre conceptuel généralement reconnu relativement à la veille stratégique. Cette section identifie certains travaux de chercheurs s'intéressant spécifiquement à la veille stratégique au Canada, sans vouloir décrire en détail les résultats de chaque étude.

Figure 2 - Cycle de la veille stratégique

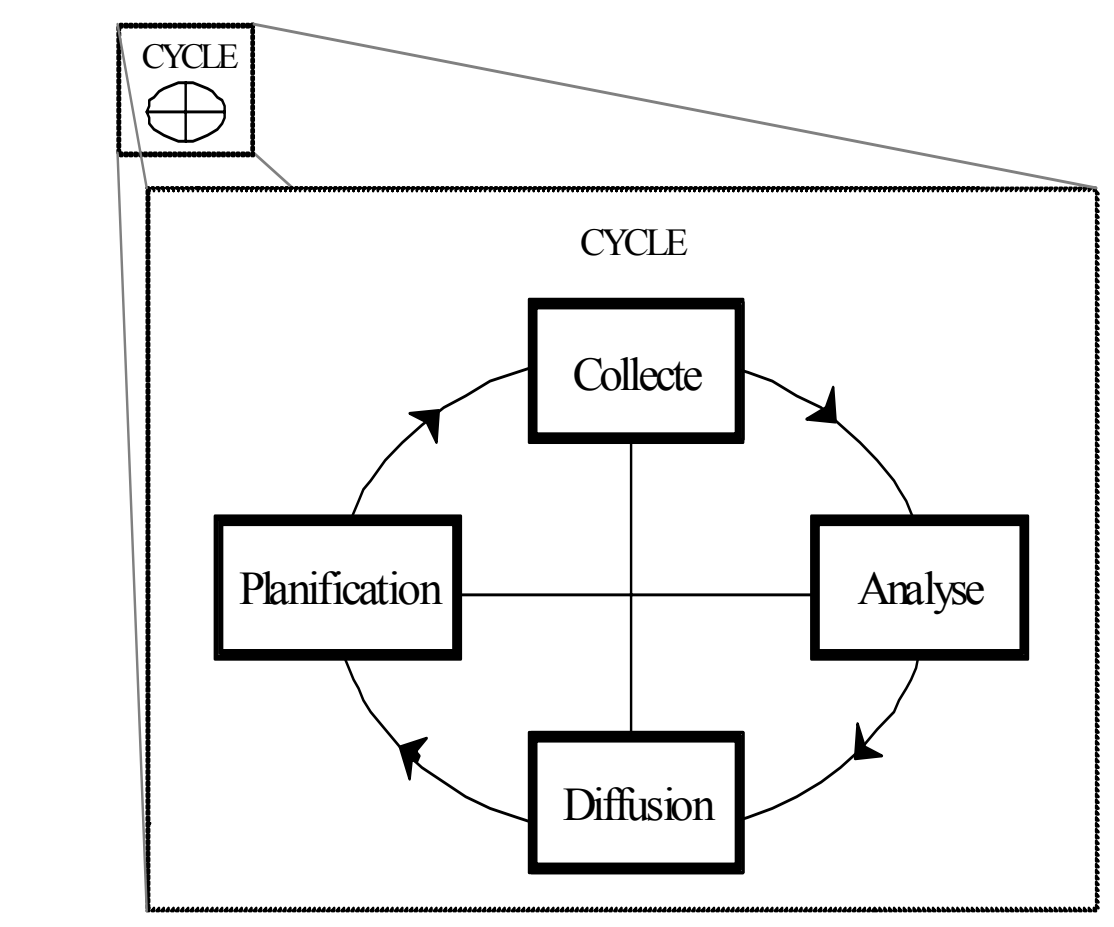

(C) 2002, François Brouard

\section{Recherches empiriques au Canada}

Les chercheurs proviennent essentiellement de deux champs disciplinaires, soit les sciences de l'information et les sciences de la gestion. Les travaux en sciences de l'information et bibliothéconomie préoccupent les chercheurs de l'Université de Toronto, de l'Université de Montréal et de l'Université McGill :

- Auster et Choo, de l'Université de Toronto, s'intéressent aux comportements de veille stratégique des chefs de la direction d'entreprises dans les secteurs des télécommunications et de l'édition.

- À l'Université de Montréal, Bergeron étudie les pratiques de veille stratégique dans de grandes organisations ainsi que les politiques gouvernementales de soutien à la veille stratégique en comparant les pratiques dans plusieurs pays. Il a d'ailleurs examiné le programme québécois des centres de veille concurrentielle.

- Bouthillier et Shearer, de 1'Université McGill, s'intéressent aux logiciels de traitement de l'information axés sur les besoins liés au processus de veille.

Les travaux en sciences de la gestion suscitent l'intérêt de chercheurs de l'Université du Québec à TroisRivières (UQTR), de l'Université Laval, des HEC de Montréal, de l'Université d'Ottawa et de l'Université Carleton :

- L’Université du Québec à Trois-Rivières constitue le pôle le plus important en veille stratégique au Canada et les chercheurs s'intéressent en particulier aux petites et moyennes entreprises (PME). Les travaux touchent la veille technologique et le développement d'outils de travail. Deux candidats 
au doctorat en administration de l'UQTR complètent actuellement leur thèse sur le thème de la veille stratégique : Dumas se penche sur l'implantation d'une cellule de veille marketing dans le secteur de l'hôtellerie et nous nous intéressons au développement d'un outil diagnostique des pratiques de veille stratégique des PME.

- Dans le cadre d'une thèse de doctorat à l'Université Laval, Audet analyse la veille stratégique chez les PME québécoises et les relations avec la performance. Deux étudiants des HEC de Montréal ont rédigé leur mémoire de maîtrise sur la veille stratégique : Dufresne examine les relations entre les activités de veille compétitive et la stratégie d'opérations dans le secteur pharmaceutique, tandis que Boucher s'intéresse aux pratiques de veille marketing dans les PME du secteur des technologies de l'information.

\section{La veille stratégique contribue notamment à améliorer la qualité des produits et services et à créer un avantage concurrentiel pour l'entreprise.}

- L'Université d'Ottawa compte sur Calof pour étudier divers aspects de la veille stratégique, autant dans les grandes entreprises et les PME que dans le secteur public. Calof a d'ailleurs participé à l'étude du CNRC-ICIST sur les pratiques de veille dans l'industrie canadienne de la technologie. Sous sa supervision, Haskins et al. ont examiné la dimension de l'éthique dans les pratiques de veille.

- L'Université Carleton compte sur l'auteur du présent article pour étudier divers aspects de la veille stratégique, en particulier les PME et le développement d'outils diagnostiques.

L'Université Carleton possède également une expertise avec un centre sur la sécurité et l'intelligence (The Canadian Centre of Intelligence and Security [CCISS]), unité de recherche de la Norman Paterson School of International Affairs (NPSIA).

\section{Publications de conseils en veille par des Canadiens}

Parmi certaines autres publications offrant un survol de conseils par des Canadiens sur des méthodes, outils et pratiques de veille stratégique, notons, à titre d'exemples, le compte rendu d'un colloque, une bibliographie, des outils et des guides pratiques. Il existe également un certain nombre de publications avec une orientation davantage professionnelle pour faire connaître les bases de la veille stratégique ou soulever certains problèmes, par exemple aux plans de la formation et de l'organisation des veilles sectorielles ou régionales.

\section{Pistes de recherche et d'action}

Compte tenu de l'état des connaissances d'un phénomène relativement nouveau, la veille stratégique doit faire l'objet d'une attention plus soutenue, notamment par une sensibilisation des dirigeants et le développement des pratiques. Par l'apprentissage, la veille stratégique favorise l'anticipation, l'action et l'innovation. La veille stratégique contribue notamment à améliorer la qualité des produits et services et à créer un avantage concurrentiel pour l'entreprise. Étant donné l'état de la connaissance relative à la veille stratégique, il y a toutefois lieu de suggérer certaines pistes de recherche et d'action ${ }^{3}$.

\section{Pistes pour les gouvernements}

- Développer une politique nationale de veille s'adressant aux entreprises.

- Investir dans le développement de la veille stratégique.

- Favoriser, avec une aide financière, l'implantation dans les entreprises de pratiques de gestion stratégique de l'information, dont la veille stratégique.

- Placer au premier plan les volets stratégiques dans le développement économique.

- Examiner les politiques publiques, notamment les règles fiscales, pouvant favoriser les pratiques de gestion, dont la veille stratégique. 
- Améliorer les systèmes d'information nationaux en dressant un inventaire du capital de connaissances des gouvernements, des collectivités et des entreprises.

- Encourager la création et le maintien des initiatives de veille stratégique (par exemple, centres de veille sectoriels ou régionaux).

- Encourager l'implantation de portail de veille.

- Recruter du personnel ayant une expertise en gestion stratégique de l'information, de la connaissance et de la veille stratégique.

- Encourager les pratiques d'étalonnage des pratiques de gestion, dont la veille stratégique.

- Fournir de l'aide financière pour les programmes de formation spécialisée destinées à améliorer les pratiques de gestion innovantes, dont la veille stratégique.

- Investir dans l'industrie de l'information et le réseau des bibliothèques.

\section{Pistes pour les universités et instituts de recherche}

- Développer des programmes de formation orientés vers la gestion et la veille stratégique.

- Modifier les programmes universitaires en gestion pour intégrer des cours portant sur la veille stratégique.

- Organiser des activités de transfert de connaissances, par exemple des conférences, des séminaires, des colloques, etc.

- Étudier les besoins et comportements informationnels des PME.

- Supporter les travaux de recherche dans le domaine de la veille stratégique.
- Promouvoir la diffusion d'information.

- Développer un cadre conceptuel de la veille stratégique.

\section{Pistes pour les entreprises}

- Participer à des activités de sensibilisation de leur personnel aux activités de veille stratégique.

- Reconnaître l'importance de développer des avantages concurrentiels.

- Communiquer constamment leurs besoins et leurs désirs aux universités et centres de recherche.

- Développer des produits et systèmes d'information après une étude des besoins.

- Reconnaître que l'information est une ressource essentielle qui fait appel à des compétences spécifiques.

- Recruter des ressources humaines compétentes en gestion de l'information.

Comme nous l'avons déjà mentionné, la veille stratégique permet à l'entreprise de mieux connaître son environnement. Selon Pateyron ${ }^{4}$ :

Seule une approche innovante, alliant veille stratégique, réflexion et imagination, en rupture par rapport aux démarches traditionnelles, peut conférer un avantage majeur: il s'agit en quelque sorte, pour l'entreprise, de redéfinir les règles du jeu et de faire évoluer les conditions du marché en sa faveur.

\section{Notes et références}

1 Membre du corps professoral à la Eric Sprott School of Business, François Brouard s'intéresse notamment, dans le cadre de ses recherches, aux PME, à l'entrepreneurship et à la veille stratégique. Il enseigne en comptabilité financière et en fiscalité. Comptable agréé de formation, il termine actuellement un doctorat en administration (DBA) à l'Université du Québec à Trois-Rivières. Sa thèse porte sur le développement d'un outil diagnostique des pratiques de 
veille stratégique des PME. François Brouard fait également partie de la nouvelle unité de recherche en entrepreneurship à la Eric Sprott School of Business, Carleton University à Ottawa.

2 Porter, M.E. (1982). Choix stratégique et concurrence, Paris, Economica, 426 p.
3 Bergeron, P. (2000). Veille stratégique et PME : comparaison des approches gouvernementales de soutien, SainteFoy, Presses de l'Université du Québec, collection Gestion de l'information, $440 \mathrm{p}$.

4 Pateyron, E. (1998). La veille stratégique, Paris, Économica, 212 p. (p. 187). 